Int. Journal of Math. Analysis, Vol. 8, 2014, no. 19, 921 - 929

HIKARI Ltd, www.m-hikari.com

http://dx.doi.org/10.12988/ijma.2014.44276

\title{
On Multiple Completeness of the System of Eigen and Associated Vectors of a Class of Operator Bundles
}

\author{
S. S. Mirzoev \\ Baku State University \\ Institute Mathematics and Mechanics of NAS of Azerbaijan \\ Baku, Ajerbaijan \\ E. B. Sultanova \\ Baku State University \\ Z. Khalilov Street 23 \\ AZ1148 Baku, Azerbaijan
}

Copyright (c) 2014 S. S. Mirzoev and E. B. Sultanova. This is an open access article distributed under the Creative Commons Attribution License, which permits unrestricted use, distribution, and reproduction in any medium, provided the original work is properly cited.

\begin{abstract}
In the paper we get the conditions on the coefficients of some class of $n$-th order operator bundle with a normal principal part, that provide $n$-fold completeness of all eigen and associated vectors of the given operator bundle. These conditions are expressed in the form of some algebraic conditions imposed on the norm of bounded operators participating in the exspression of the operator bundle.
\end{abstract}

In a separable Hilbert space $H$ consider an $n$-th order polynomial operator bundle

$$
L(\lambda)=E-\lambda^{n} C^{n}+\sum_{j=0}^{n-1} \lambda^{j}\left(B_{j}+K_{j}\right) C^{j}
$$


where $\lambda$ is a spectral parameter, $E$ is a unit operator, and the other coefficients satisfy the conditions:

1. $C$ is a complete (i.e. from the condition $C x=0$ it follows $x=0$ ) completely normal operator whose spectrum is contained in the conditional sector

$$
S_{\varepsilon}=\{\lambda:|\arg \lambda| \leq \varepsilon\}, \quad 0 \leq \varepsilon<\frac{\pi}{n}
$$

2. The operators $B_{j}$ are bounded in $H$, i.e. $B_{j} \in L(H), j=\overline{0, n-1}$;

3. The operators $K_{j}$ are completely continuous in $H$, i.e. $K_{j} \in \sigma_{\infty}(H)$.

Not that M.V.Keldysh has introduced the notion of $n$-fold completeness of the system of eigen and associated vectors of an operator bundle [1] and proved a theorem on $n$-fold completeness of this system in $H$ in the sense of M.V.Keldysh for $C=C^{*}>0, C \in \sigma_{\infty}, B_{j}=0, K_{j} \in \sigma_{\infty}(H)[1,2]$. In the sequel, when $C$ is a complete normal operator, $C^{n}$ is a self-adjoint operator, $C \in \sigma_{\infty}, B_{j}=0, K_{j} \in \sigma_{\infty}(H)$, the bundle (1) was investigated in [3]. When $C \in \sigma_{\infty}, C$ is a normal operator whose spectrum is contained in a finitely many rays emanating from the origin or coordinates for $B_{j}=0, K_{j} \in \sigma_{\infty}(H)$, the $n$-fold completeness of the system of eigen and associated vectors in the sense of M.V.Keldysh was studied in [4]. The operator bundle (1) for $n=2$ was investigated in $[5,7,8]$. It should be noted that at different situations, the operator bundle (1) for $C=C^{*}>0$ was considered for example, in the papers [5-11]. For $n=2$, the operator bundle (1) for $K_{j}=0(j=\overline{0,1})$ was considered in the paper [6], when the operator coefficients are unbounded operators, and a theorem on the double completeness of the system of eigen and associated vectors responsible for boundary value problems on the finite segment was proved.

Definition 1. If for $\lambda \in C$ (here $C$ is a complex plane), the operator bundle (1) is invertible, i.e. these exists $L^{-1}(\lambda)$, is bounded and defined on all of the space $H$, then $\lambda$ is said to be a regular point of $L(\lambda)$, and $L^{-1}(\lambda)$ is a resolvent of the operator bundle $L(\lambda)$.

Definition 2. If for $\lambda_{0} \in C$ the equation $L\left(\lambda_{0}\right) x=0$ has the solution $x_{0} \neq 0$, then $\lambda_{0}$ is called a characteristic number of $L(\lambda)$, and $x_{0}$ an eigen vector of the bundle responsible for the number $\lambda_{0}$. If the system $\left\{x_{0}, x_{1}, \ldots, x_{m}\right\}$ satisfies the equations

$$
\left.\sum_{j=0}^{h} \frac{L^{j}(\lambda)}{j !}\right|_{\lambda=\lambda_{0}} x_{h-j}, \quad h=\overline{0, m}
$$


then this system is said to be the system of eigen and associated vectors of the bundle $L(\lambda)$.

Obviously, several series of the system of eigen and associated vectors may be responsible for the eigen vector $x_{0}$. We'll denote them by $\left\{x_{0, j, 0}, x_{0, j, 1}, \ldots, x_{0, j, m_{0 j}}\right\}$, $j=\overline{1, q_{0}}$. The number $m_{0 j}+1$ is called the length of the system, $\max _{j} m_{0 j}+1$ is the multiplicity of the characteristic number $\lambda_{0}$.

Definition 3. If the operator bundle $L(\lambda)$ has a spectrum consisting only of characteristic numbers with finite multiplicity, we say that $L(\lambda)$ has a discrete spectrum.

Definition 4. If $K \in \sigma_{\infty}(H)$, then the eigen numbers of the operator $\left(K^{*} K\right)^{1 / 2}$ are called $s$-numbers of the operator $K$. If for some $\rho>0$ the series $\sum_{n=1}^{\infty} s_{n}^{\rho}<\infty$, then it is said that $K \in \sigma_{\rho}(H)$.

The following lemma indicates when $L(\lambda)$ has a discrete spectrum.

Lemma 1. Let conditions 1)-3) be fulfilled. If the operator $E+B_{0}$ is inversible in $H$, then the bundle $L(\lambda)$ has a discrete spectrum with a unique limit point at infinity.

Proof. Represent the operator bundle in the form

$$
L(\lambda)=L_{0}(\lambda)+B(\lambda)+K(\lambda)
$$

where

$$
L_{0}(\lambda)=E-\lambda^{n} C^{n}, B(\lambda)=\sum_{j=0}^{n-1} \lambda^{j} B_{j} C^{j}, \quad K(\lambda)=\sum_{j=0}^{n-1} \lambda^{j} K_{j} C^{j} .
$$

Then it is obvious that

$$
\begin{gathered}
L(\lambda)=E-\lambda^{n} C^{n}+\sum_{j=1}^{n-1} \lambda^{j} B_{j} C^{j}+B_{0}+K(\lambda)= \\
=\left(E+B_{0}\right)\left(E-\lambda^{n}\left(E+B_{0}\right)^{-1} C^{n}+\sum_{j=1}^{n-1} \lambda^{j}\left(E+B_{j}\right)^{-1}\left(B_{j}+K_{j}\right) C^{j}+K_{0}\right) .
\end{gathered}
$$

Without loss of generality we can assume that $E+K_{0}$ is invertible in $H$, in the coutrary case, changing $\lambda$ by $\lambda+\delta$, we can choose $\delta$ so that the free term would be inversible. Thus,

$$
L(\lambda)=\left(E+B_{0}\right)(E+Q(\lambda)),
$$

where

$$
Q(\lambda)=\left(-\lambda^{n}\left(E+B_{0}\right)^{-1} C^{n}+\sum_{j=1}^{n-1} \lambda^{j}\left(E+B_{j}\right)^{-1}\left(B_{j}+K_{j}\right) C^{j}+K_{0}\right) .
$$


Obviously, $Q(\lambda) \in \sigma_{\infty}(H)$ for any $\lambda \in C$ and $E+Q(0)=E+K_{0}$ is inversible in $H$, then by the Keldysh lemma [2], the operator bundle $E+Q(\lambda)$ has a discrete spectrum with a unique limit point at infinity. From the representation

$$
L(\lambda)=\left(E+B_{0}\right)(E+Q(\lambda))
$$

we get that the statement of the lemma is true.

Lemma 2. Let conditions 1)-3) be fulfilled, the operator $E+B_{0}$ be inversible in $H$, and $C \in \sigma_{\rho}(H), 0<\rho<\infty$. Then the resolvent $L^{-1}(\lambda)$ is represented in the form of ratio of two entire functions of order $\rho$ and of minimal type at order $\rho$.

Proof. Since the coefficients of the operator bundle are completely continuous, $\left(E+B_{0}\right)^{-1}$ is a bounded operator in $H$, then from the condition $C \in C_{\rho}(H)$ it follows that $C^{j} \in \sigma_{\rho / j}(H)$, therefore the coefficient $\lambda^{n}$ equals $\left(E+B_{0}\right)^{-1} C^{n} \in$ $\sigma_{\rho / j}(H)$. The coefficient $\lambda^{j}$ is the operator $\left(E+B_{0}\right)^{-1}\left(B_{j}+K_{j}\right) C^{j} \in \sigma_{\rho / j}(H)$, then it follows from the Keldysh lemma [2] that $(E+Q(\lambda))^{-1}$ is represented in the form of ratio of two entire functions of order less than order $\max _{j}\left(j, \frac{\rho}{j}\right)=\rho$ and of minimal type at order $\rho$. From representation (2) we get

$$
L^{-1}(\lambda)=\left(E+B_{0}\right)^{-1}(E+Q(\lambda))^{-1} .
$$

Therefore, $L^{-1}(\lambda)$ also possesses this property. The lemma is proved.

Now prove a theorem on estimation of the resolvent.

Theorem 1. Let conditions 1)-2) be fulfilled, and it hold the inequality

$$
q(\varepsilon)=\sum_{j=0}^{n-1} d_{j}(\varepsilon)\left\|B_{j}\right\|<1,
$$

where

$$
\begin{gathered}
d_{0}(\lambda)= \begin{cases}1, & \varepsilon \in\left(0, \frac{\pi}{2}\right] \\
\frac{1}{\sin n \varepsilon}, & \varepsilon \in\left(\frac{\pi}{2 n}, \frac{\pi}{n}\right)\end{cases} \\
d_{j}(\varepsilon)=\frac{1}{\cos \frac{\pi \varepsilon}{2}} d_{n, j} ; \quad d_{n, j}=\left(\frac{j}{n}\right)^{\frac{j}{n}}\left(\frac{n-j}{n}\right)^{\frac{n-j}{n}}, j=\overline{1, n-1} .
\end{gathered}
$$

Then on the rays $\Gamma_{m}=\left\{\lambda: \arg \lambda=\frac{2 \pi m}{n}+\frac{\pi}{n}\right\}, m=\overline{0, n-1}$, and the operator bundle

$$
M(\lambda)=E-\lambda^{n} C^{n}+\sum_{j=0}^{n-1} \lambda^{j} B_{j} C^{j}
$$

is inversible, moreover, $\left\|M^{-1}(\lambda)\right\| \leq$ const, $\lambda \in \Gamma_{m}$. 
Proof. Let $\lambda \in \Gamma_{m}, . . \lambda=r e^{i\left(\frac{2 \pi m}{n}+\frac{\pi}{n}\right)}$. Then

$$
\begin{aligned}
M(\lambda)= & E-r^{n} e^{i(2 \pi m+\pi)} C^{n}+\sum_{j=0}^{n-1} r^{j} e^{i\left(\frac{2 \pi m}{n}+\frac{\pi}{n}\right) j} B_{j} C^{j}= \\
& =E+r^{n} C^{n}+\sum_{j=0}^{n-1} r^{j} e^{i\left(\frac{2 \pi m}{n}+\frac{\pi}{n}\right) j} B_{j} C^{j} .
\end{aligned}
$$

Obviously, $E+r^{n} C^{n}$ is inversible in $H$, since the spectrum of the operator $C^{n}$ is contained in the angular sector $\{\lambda:|\arg z| \leq n \varepsilon\}, 0 \leq \varepsilon<\frac{\pi}{n}$ and $n \varepsilon<n \frac{\pi}{n}=\pi$, i.e. $-\frac{1}{r^{n}} \notin \sigma\left(C^{n}\right)$. Thus, for $\lambda \in \Gamma_{m}$

$$
M(\lambda)=\left(E+\sum_{j=0}^{n-1} r^{j} e^{i\left(\frac{2 \pi m}{n}+\frac{\pi}{n}\right) j} B_{j} C^{j}\left(E+r^{n} C^{n}\right)^{-1}\right)\left(E+r^{n} C^{n}\right) .
$$

Since

$$
\left\|\sum_{j=0}^{n-1} r^{j} e^{i\left(\frac{2 \pi m}{n}+\frac{\pi}{n}\right)} B_{j} C^{j}\left(E+r^{n} C^{n}\right)\right\| \leq \sum_{j=0}^{n-1}\left\|B_{j}\right\|\left\|r^{j} C^{j}\left(E+r^{n} C^{n}\right)^{-1}\right\|,
$$

then we estimate the norm $\left\|r^{j} C^{j}\left(E+r^{n} C^{n}\right)\right\|, j=\overline{0, n-1}$. Since $C$ is a complete normal operator and its spectrum $\xi_{k}=\mu_{k} e^{i \varphi_{k}}, \mu_{k}>0,\left|\varphi_{k}\right|<\varepsilon$, then

$$
\begin{gathered}
\left\|\sum_{j=0}^{n-1} r^{j} e^{i\left(\frac{2 \pi m}{n}+\frac{\pi}{n}\right) j} B_{j} C^{j}\left(E+r^{n} C^{n}\right)\right\|=\sup _{\xi_{k} \in \sigma(A)}\left|r^{j} \xi_{k}^{j}\left(E+r^{n} \xi_{k}^{n}\right)^{-1}\right|= \\
=\sup _{\mu_{k}>0,\left|\varphi_{k}\right|<\varepsilon}\left|r^{j} \mu_{k}^{j}\left(1+r^{n} \mu_{k}^{n} e^{i n \varphi_{k}}\right)^{-1}\right|= \\
=\sup _{\mu_{k}>0,\left|\varphi_{k}\right|<\varepsilon}\left|r^{j} \mu_{k}^{j}\left(1+r^{2 n} \mu_{k}^{2 n}+2 r^{n} \mu^{n} \cos n \varphi_{k}\right)^{-1 / 2}\right| \leq \\
\leq \sup _{\mu_{k}>0}\left|r^{j} \mu_{k}^{j}\left(1+r^{2 n} \mu_{k}^{2 n}+2 r^{n} \mu^{n} \cos n \varepsilon\right)^{-1 / 2}\right| .
\end{gathered}
$$

Let $j=0$. If $\cos n \varepsilon \geq 0\left(0<\varepsilon \leq \frac{\pi}{2 n}\right)$, then

$$
\left(1+r^{2 n} \mu_{k}^{2 n}+2 r^{n} \mu^{n} \cos n \varepsilon\right)^{-1 / 2} \leq\left(1+r^{2 n} \mu_{k}^{2 n}\right)^{-1 / 2} \leq 1,
$$


and for $\cos n \varepsilon<0\left(\frac{\pi}{2 n}<\varepsilon \leq \frac{\pi}{n}\right)$

$$
\left(1+r^{2 n} \mu_{k}^{2 n}+2 r^{n} \mu^{n} \cos n \varepsilon\right)^{-1 / 2} \leq \frac{1}{\sin n \varepsilon}
$$

since the function $\left(1+\tau^{2}+2 \tau \cos n \varepsilon\right)^{-1}$ accepts its maximum value for $\tau=$ $-\cos n \varepsilon>0$ equal $\frac{1}{\sin n \varepsilon}$. Thus,

$$
\left\|\left(E+r^{n} C^{n}\right)^{-1}\right\| \leq d_{0}(\varepsilon) .
$$

Now let's consider $j=\overline{1, n-1}$. Obviously,

$$
\begin{gathered}
\left|r^{j} \mu_{k}^{j}\left(1+r^{2 n} \mu_{k}^{2 n}+2 r^{n} \mu^{n} \cos n \varepsilon\right)^{-1 / 2}\right| \leq \\
\leq\left|r^{j} \mu_{k}^{j}\left(\left(1+r^{n} \mu_{k}^{n}\right)^{2}\left(1-2 r^{n} \mu^{n} \frac{1-\cos n \varepsilon}{\left(1+r^{n}\right)^{2}}\right)\right)^{-1 / 2}\right| \leq \\
\leq\left|r^{j} \mu_{k}^{j}\left(\left(1+r^{n} \mu_{k}^{n}\right)^{-1}\left(1-\frac{4 r^{n} \mu_{k}^{n} \sin ^{2} \frac{n \varepsilon}{2}}{4 r^{n} \mu_{k}^{n}}\right)^{-1 / 2}\right)\right|= \\
=\left|r^{j} \mu_{k}^{j}\left(1+r^{n} \mu_{k}^{n}\right)^{-1}\left(\cos \frac{n \varepsilon}{2}\right)^{-1}\right| \leq \\
\leq \frac{1}{\cos \frac{n \varepsilon}{2}} \sup _{\tau>0}\left|\tau^{j}\left(1+\tau^{n}\right)^{-1}\right|=\left(\frac{j}{n}\right)^{\frac{j}{n}}\left(\frac{n-j}{n}\right)^{\frac{n-j}{n}} \frac{1}{\cos \frac{n \varepsilon}{2}}=d_{j}(\varepsilon) .
\end{gathered}
$$

Consequently, for $j=\overline{1, n-1}$

$$
\left\|r^{j} \mu_{k}^{j}\left(E+r^{n} C^{n}\right)\right\| \leq d_{j}(\varepsilon)
$$

Taking into account inequalities (7), (8) in inequality (5), we get:

$$
\sum_{j=0}^{n-1}\left\|B_{j}\right\|\left\|r^{j} C^{j}\left(E+r^{n} C^{n}\right)^{-1}\right\| \leq \sum_{j=0}^{n-1} d_{j}(\varepsilon)\left\|B_{j}\right\|=q_{j}(\varepsilon)<1 .
$$

Then the operator bundle $M(\lambda)$ is inversible on the rays $\Gamma_{m}$

$$
M^{-1}(\lambda)=\left(E-\lambda^{n} C^{n}\right)^{-1}\left(E+B(\lambda)\left(E-\lambda^{n} C^{n}\right)^{-1}\right)^{-1}
$$


and

$$
\left\|M^{-1}(\lambda)\right\|=\left\|\left(E-\lambda^{n} C^{n}\right)^{-1}\right\| \frac{1}{1-q(\varepsilon)}=d_{0}(\varepsilon)(1-q(\varepsilon))^{-1}=\text { const. }
$$

The theorem is proved.

Corollary. Let the conditions of theorem 1 and conditions 3) be fulfilled. Then on the rays $\Gamma_{m}(m=\overline{0, n-1})$ for large $|\lambda|$ there exists the resolvent $L^{-1}(\lambda)$, moreover, $\left\|L^{-1}(\lambda)\right\| \leq$ const $, \lambda \in \Gamma_{m},|\lambda|>R_{0}$.

Proof. For $\lambda \in \Gamma_{m}$

$$
L(\lambda)=M(\lambda)+K(\lambda)=\left(E+K(\lambda) M^{-1}(\lambda)\right) M(\lambda),
$$

and

$$
K(\lambda) M^{-1}(\lambda)=K(\lambda)\left(E-\lambda^{n} C^{n}\right)^{-1}\left(E+B(\lambda)\left(E-\lambda^{n} C^{n}\right)^{-1}\right)^{-1} .
$$

Since

$$
K(\lambda)\left(E-\lambda^{n} C^{n}\right)^{-1}=\sum_{j=0}^{n-1} \lambda^{j} K_{j} C^{j}\left(E-\lambda^{n} C^{n}\right)^{-1},
$$

then using the M.V.Keldysh lemma, we get that for $K_{j} \in \sigma_{\infty}(H)$

$$
\lim _{|\lambda| \rightarrow \infty, \lambda \in \Gamma_{m}}\left\|\lambda^{j} K_{j} C^{j}\left(E-\lambda^{n} C^{n}\right)^{-1}\right\|=0 .
$$

Therefore, for $\lambda \in \Gamma_{m}$ and for large $|\lambda|$ the norm $\left\|K(\lambda)\left(E-\lambda^{n} C^{n}\right)^{-1}\right\|$ is a rather small number. Then we get that for large $R_{0}>0 E+K(\lambda) M^{-1}(\lambda)$ is inversible in $H$ and $L^{-1}(\lambda)=M^{-1}(\lambda)\left(E+K(\lambda) M^{-1}(\lambda)\right)^{-1}$. Hence it follows that for $\lambda \in \Gamma_{m},|\lambda|>R_{0}$

$$
\left\|L^{-1}(\lambda)\right\|=\text { const }\left\|M^{-1}(\lambda)\right\| \leq \text { const. }
$$

The corollary is proved.

Now give the definition of $n$-fold completeness of the system of eigen and associated vectors and prove the main theorem.

Definition 5. Let $\lambda_{i}$ be a characteristic number of $L(\lambda)$ and $\left\{x_{i, j, 0}, x_{i, j 1}, \ldots, x_{i j, m_{i j}}\right\}$, $i=\overline{1, \infty}, q=\overline{1, m_{i}}$ be the system of eigen and associated vectors responsible for $\lambda_{i}$. Then the vector-function

$$
u_{i, j, h}(t)=e^{\lambda_{i} t}\left(\frac{t^{h}}{h !} x_{i, j, 0}+\frac{t^{h-1}}{(h-1) !} x_{i, j, 1}+\ldots+x_{i j, h}\right), h=\overline{0, m_{i j}}, j=\overline{1, q_{i}}
$$

satisfies the equation $L(d / d t) u(t)=0$ and is said to be elementary solutions of the homogeneous equation $L(d / d t) u(t)=0$. In the space $H^{n}=H \oplus H \oplus$ $\ldots \oplus H$ consider the system

$$
K=\left\{\left(u_{i, j h}(0), u_{i j h}^{\prime}(0), \ldots, u_{i, j h}^{(n-1)}(0)\right)\right\}_{i=1, h=\overline{0, m_{i j}}, j=\overline{1, q_{i}}}^{\infty} .
$$


If the system $K$ is complete in the space $H^{n}$, we say that the system of eigen and associated vectors of the bundle $L(\lambda)$ is $n$-fold complete in $H$ in the sense of M.V.Keldysh.

It holds

Theorem 2. Let the conditions of theorem $1 K_{j} \in \sigma_{\infty}$ and $C^{-1} \in \sigma_{\rho}(H)$ be fulfilled, for $0<\rho \leq \frac{n}{2}$. Then system of eigen and associated vectors of the bundle $L(\lambda)$ be $n$ fold complete in $H$ in the sense of M.V.Keldysh.

Proof. From the results of [2] it follows that if $K$ is not $n$-fold complete system in $H$, then there exist the vectors $f_{i} \in H, i=\overline{0, n-1}$ such that $\sum_{i=0}^{n-1}\left\|f_{i}\right\| \neq 0$ and the vector-function

$$
R(\lambda)=\left(R^{-1}(\bar{\lambda})\right)^{*}\left(f_{0}+\lambda_{1} f_{1}+\ldots+\lambda^{n-1} f_{n-1}\right)
$$

is an entire function, is of order $\rho$, and has a minimal type at order $\rho$. Since on the rays $\Gamma_{m}$, by theorem $1, R(\lambda)$ is no faster than $|\lambda|^{n-1}$, then taking into account that the angle between the neighboring rays $\Gamma_{m}$ equals $\frac{2 \pi}{n}=\frac{\pi}{n / 2}$, then from the Fragmen-Lindeloff theorem it follows that

$$
R(\lambda)=g_{0}+\lambda g_{1}+\ldots+\lambda^{n-1} g_{n-1} .
$$

Hence it follows that

$f_{0}+\lambda f_{1}+\ldots+\lambda^{n-1} f_{n-1}=\left(E-\lambda^{n} C^{* n}+B^{*}(\lambda)+K^{*}(\lambda)\right)\left(g_{0}+\lambda g_{1}+\ldots+\lambda^{n-1} g_{n-1}\right)$.

Here, making comparison of the coefficients for $\lambda^{n-1}$, we get $C^{* n} g_{n-1}=0$. Since $C$ is a complete operator, then $g_{n-1}=0$. Then, making comparison of the coefficients $\lambda^{2 n-2}$, we get $g_{n-2}=0$. In the same way we get $g_{i}=$ $0(i=\overline{0, n-1})$. Then $f_{0}+\lambda f_{1}+\ldots+\lambda^{n-1} f_{n-1}=0$, i.e. $f_{0}=f_{1}=\ldots=$ $f_{n-1}=0$. And this contradicts the condition $\sum_{i=0}^{n-1}\left\|f_{i}\right\| \neq 0$. The theorem is proved.

\section{REFERENCES}

[1] M.V.Keldysh. On eigenvalues and eigen functions of some class of not self-adjoint equations. DAN SSSR, 1951, v. 77, No1, p.11-41.(Russian)

[2] M.V.Keldysh. On completeness of eigen functions of some classes of not self-adjoint linear operators. Uspekhi mat. nauk, 1971, v.27, issue 4, p. 15-47. (in Russian)

[3] V.I.Matsayev, E.I.Mogulskii. Some indications of multiple completeness of eigen and associated vectors of polynomial boundles of operators. Theory of function, functional analysis and their applications. Kharkov, v.13, 1971, p.3-45. (in Russian)

[4] G.V.Radzievskii. A problem on completeness of the root vectors in spectral theory of operator-functions. Uspekhi mat. nauk, 1982, v.37, issue 2, p. 81145. (Russian) 
[5] Sultanova E.B. On spectral theory of quadratic operator pensil. Transac. of NAS of Azerb., 2013, v. 33, No4, p.123-128.

[6] S.S.Mirzoev, M.V.Karaaslan, R.Z.Gumbataliev. To theory of second order operator-differential equations. Doklady RAN, 2013, v.453, No6, p.610-612. (in Russian)

[7] E.B.Sultanova. On completeness of eigen and associated vectors of a class of operator boundles. Vestnik Pedagogicheskogo Universita, 2013, No3, p.43-46. (in Russian)

[8] Mirzoev S.S., Salimov M.Yu. Completeness of elementary solutions to a class of second order operator differential equations. Sib. Math. J., 2010, t.51, No4, p.815-828. (in Russian)

[9] S.S.Mirzoev, R.Z.Gumbataliev. On completeness of the system of elementary solutions of a class of operator-differential equation on a finite interval. Doklady RAN, 2010, v.431, No4, p.454-456. (in Russian)

[10] Gumbataliev R.Z. On completeness of elementary generalized solutions of a class of operator-differential equations of a higher order. Turkish Journal of Mathematics, 2009, v.33, No4, pp.383-396.

Received: April 5, 2014 